\title{
Development of a power supply for multi-angle electric impedance tomography complex
}

\author{
Grayr K. Aleksanyan ${ }^{1,}$, Ivan D. Shcherbakov ${ }^{1}$, Artem I. Kucher ${ }^{1}$, Evgeniy A. Volchenkov ${ }^{2}$ \\ ${ }^{1}$ Department of Information and Measuring Systems and Technologies, Platov South-Russian State \\ Polytechnic University (NPI), 346428 Novocherkassk, Russia \\ ${ }^{2}$ Federal state educational institution of higher education "Rostov Institute of Law of the Ministry of \\ Internal Affairs of the Russian Federation", Volgodonsk branch, 347360 Volgodonsk, Russia
}

\begin{abstract}
In the present work, the development of a multi-channel power supply for the hardware part of the hardware-software complex of multiangle electric impedance tomography is considered. The requirements for the developed module are considered based on the selected hardware components, as well as the patient and medical personnel safety requirements, taking into account the standards developed by the International Electrotechnical Commission. Taking into account the formulated requirements, a structural, electrical schematic diagram of the power supply was developed, as well as a printed circuit board and an assembly drawing, and a prototype model was built. The issues of patient and medical personnel safety during the research using the multi-angle electric impedance tomography method with the use of the developed module are considered, including in the case of joint use with electrosurgical devices or defibrillators.
\end{abstract}

\section{Introduction}

As mentioned in [1], the current source is one of the central components for multi-angle electric impedance tomography (MMEIT) devices [2-4], but the power source plays an equally important role, the final stability and accuracy of the measurements also depend on the stability of the parameters.

With the use of power supplies in medical devices and apparatus, in addition, the electrical safety of the patient, medical personnel and equipment during the study, including the joint use of the hardware-software complex MMEIT (HSC MMEIT) and other devices, affecting the patient with the help of an electric current.

Thus, the most important parameters for choosing the circuit-based solution of the current source for the MMEIT hardware and software complex is electrical safety and ensuring the stability of the necessary parameters of supply voltages.

*Corresponding author: graer@yandex.ru 


\section{Ensuring the required parameters of supply voltages}

As shown in the hardware structure diagram of the hardware-software complex MMEIT [5], the power supply is connected to several $P_{n}$ units, which require a set of various supply voltages $U_{p I}$ for operation, in addition, the source of each $U_{P I}$ must provide the current consumption of the $I_{P I}$ of all consumers connected to it.

Thus, the power supply for the hardware part of the HSC MMEIT should be a set of modules, each of which provides $U_{p I}$ and $I_{p I}$ in the corresponding channel $i$, to which consumers are connected.

The block diagram of the described multi-channel power supply is shown in Figure 1.

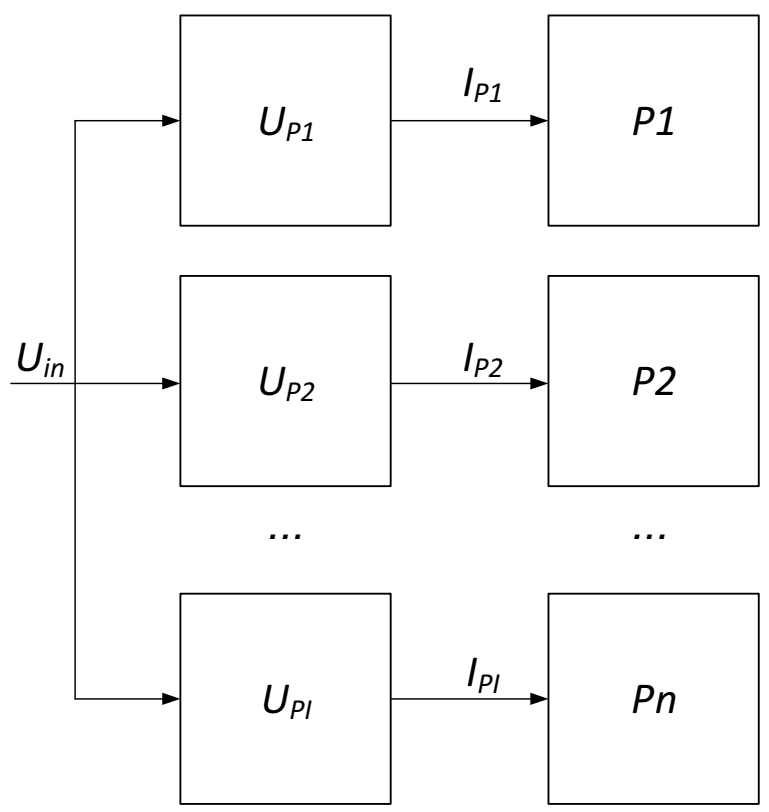

Fig. 1. Block diagram of the described multi-channel power source

As can be seen from the presented diagram, each of the channels of power supply $i$ is connected to $P_{n}$, providing the required voltage $U_{p I}$.

\section{Ensuring electrical safety requirements}

As stated in [6], safety is a key element in the design of electronic equipment, especially important in the design of medical equipment. Given that for healthy patients, and especially for patients, even small leakage currents is inadmissible. Special requirements for the electrical safety of medical equipment are regulated by international standards. Thus, the standard IEC 60601-1 [7] regulates the general requirements for the safety of medical devices taking into account their functional characteristics. According to the standard classification, the device for electrical impedance tomography refers to a product of class II type BF (not intended for direct application to the heart), that is, additional (reinforced) insulation should be provided, in the absence of protective earthing. Additional insulation can be provided by galvanic isolation, DC-DC insulators can be used for this, both up-and-down and voltage-reducing insulators. The standard implies the protection of medical devices with a potential difference of at least $5000 \mathrm{~V}$ for the working part of 
products with defibrillator protection, so for a selected set of supply voltages it is possible to use DC-DC converters with a breakdown voltage of at least $6300 \mathrm{~V}$.

A separate problem of ensuring electrical safety is galvanic isolation of data transmission lines, which unites the hardware of the complex and a personal computer that conducts mathematical calculations. Given the wide dissemination of the USB data transfer standard, the most suitable is the compact highly integrated iCoupler solution from Analog Devices [8], which allows up to 6 high-speed data lines based on transformer galvanic isolation, certified for medical use without the limitations inherent in traditional optocoupler solutions: complex electrical circuits with a large number of components, increased energy consumption, the need to balance the favorable temperature regime of operation and an acceptable data rate.

\section{Implementation of selected solutions}

Thus, the solution based on DC-DC converters and galvanic decoupling of data lines simultaneously solves several problems:

- galvanic isolation of the patient circuit and the supply circuit;

- protection of the personal computer connected to the hardware [9], in case of joint use of the AIC MIET and a defibrillator or an electrosurgical high-frequency apparatus;

- the effect of increasing the noise immunity of the system is also achieved.

The block diagram of the power supply with galvanic isolation of power lines and data transmission is shown in Fig. 2.
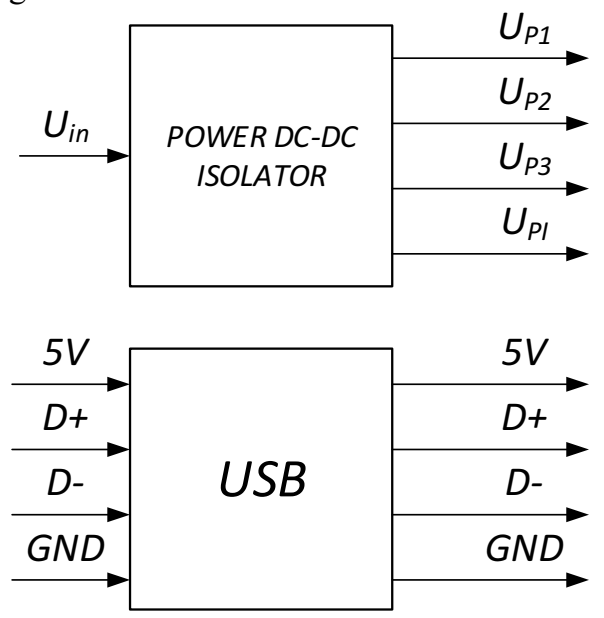

Fig. 2. Block diagram of the power source with galvanic isolation of power lines and data transmission.

On the basis of the structural diagram, an electrical schematic circuit is developed, shown in Figure 3. It uses as an input voltage for all DC-DC converters a 5V USB channel, a bipolar power supply for analog circuits, and a $5 \mathrm{~V}$ line for digital circuits. Galvanic isolation of USB data transmission lines is also provided on the basis of the iCoupler Analog Devices solution, with a guaranteed breakdown voltage of at least $5 \mathrm{kV}$ isolation. 


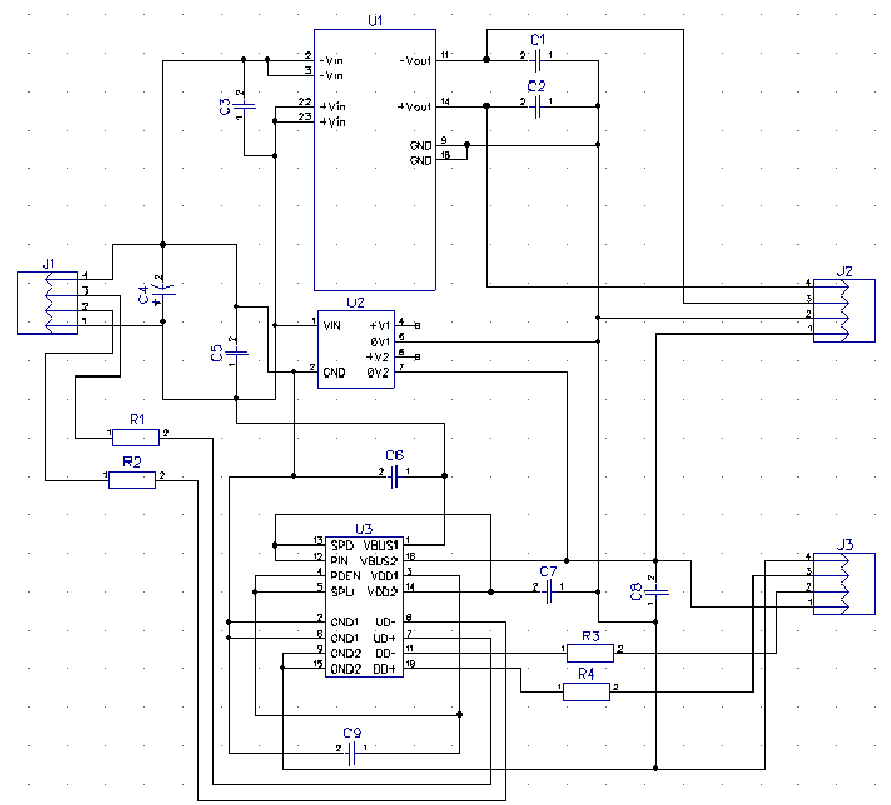

Fig. 3. Power supply with galvanic isolation of data lines. Electric schematic diagram.

Based on the developed electrical circuit, a prototype was built (shown in Fig. 4 (a)), which showed the effectiveness of the selected solutions, as well as the printed circuit board (shown in Fig. 4 (b, c)).

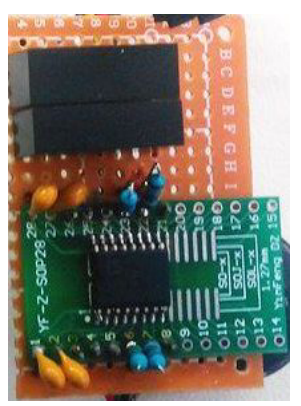

a)

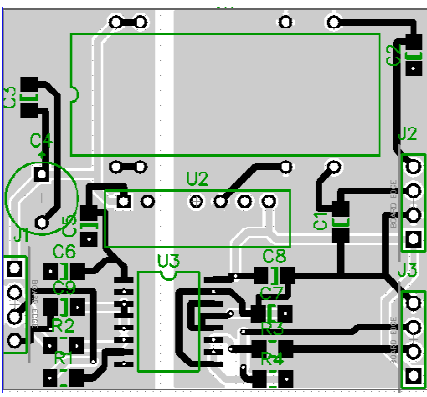

b)

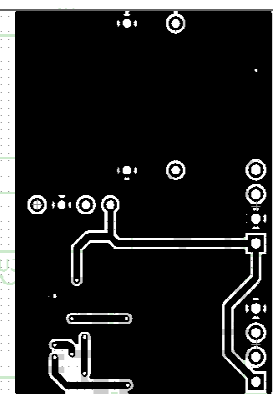

c)

Fig. 4. Power supply with galvanic isolation a) model sample, b) printed circuit board, top view, printed circuit board, bottom view.

When designing a printed circuit board, special attention was paid to the thickness of the power conductors in order to reduce the losses on their electrical resistance $[10,11]$, in addition, screening was developed at the bottom of the printed circuit board to improve the noise immunity of the entire device $[12,13]$. The shielding is divided for the input and isolated parts of the power supply in accordance with the norms of the gap width [4] to prevent the occurrence of leakage currents as a result of its breakdown. Polygons of the printed circuit board providing shielding are connected to common points of the input and isolated parts of the current source, respectively. 


\section{Conclusion}

The role of the power supply as part of the hardware-software complex of multi-angle electric impedance tomography is considered. Problems that arise when designing current sources for medical equipment, requirements for them are considered. Separate consideration is given to the safety requirements of the patient and medical personnel, taking into account international standards, including in the case of joint use with electrosurgical devices or defibrillators.

Solutions are found that meet all of the above requirements. A structural, electrical schematic diagram of the power source was developed, as well as a printed circuit board, and a model prototype was built.

Work is performed within the grant of President of Russian Federation for state support of young Russian scientists MK-196.2017.8 "Development of theoretical foundations and algorithms for multiview systems are three-dimensional electrical impedance tomography for non-invasive medical imaging".

\section{References}

1. G. K. Aleksanyan, I. D. Shcherbakov, A. I. Kucher, Jour. Eng. Appl. Sc., 12(3), 587 (2017)

2. G. K. Aleksanyan, N. I. Gorbatenko, V. V. Grechikhin, T. N. Phong, T. D. Lam, ARPN J. of Eng. and Appl. Sc., 11(9), 5871 (2016)

3. D. V. Shaykhutdinov, N. I. Gorbatenko, G. K. Aleksanyan, V. V. Grechikhin, K. M. Shirokov, V. I. Dubrov, M. V. Lankin, Res. J. of Appl. Sc., 10, 173 (2015)

4. G. K. Aleksanyan, I. D. Shcherbakov, A. I. Kucher, V. V. Demyanov, J. of Eng. and Appl. Sc., 12(8), 2129 (2017)

5. G. K. Aleksanyan, N. I. Gorbatenko, A. D. Tarasov, Res. Jour. Appl. Sc., 9 (12), 1030 (2014)

6. V. K. Zhdankin, El. comp., 2, 79 (2006)

7. IEC 60601-1-11:2015. Info: https://www.iso.org/standard/65529.html (2015)

8. iCoupler ${ }^{\circledR}$ Technology. Info: http://www.analog.com/en/products/landingpages/001/icoupler-technology-alternative-to-optocouplers.html (2012)

9. G. K. Aleksanyan, I. D. Shcherbakov, A. I. Kucher, A. V. Sulyz, AIP Conf. Proc., 1952, 020105 (2018)

10. A. P. Nenashev, Designing of radio-electronic tools (Vysshaya Shkola, Moscow, 1990)

11. G. D. Frumkin, Calculation and design of REE, 523 (Vysshaya Shkola, Moscow, 1989)

12. M. E. Osinkina, V.P. Kismereshkin, ONV, 149 (5), 115 (2016)

13. D. Ardizzoni Comp. and Techn., 77, 157 (2007) 